\title{
ConseCuencias de la domesticación de Stenocereus STELLATUS EN EL TAMAÑO DE LAS SEMILLAS Y EN LA GERMINACIÓN EN UN GRADIENTE DE ESTRÉS HÍDRICO
}

\author{
Juana Rodríguez-Morales, Susana Guillén ${ }^{1}$ y Alejandro Casas \\ Centro de Investigaciones en Ecosistemas, Morelia, Michoacán, México \\ ${ }^{1}$ Autor para la correspondencia: susana.guillen@st.ib.unam.mx
}

\begin{abstract}
Resumen: Stenocereus stellatus es una cactácea columnar de importancia ecológica y cultural en el Valle de Tehuacán; sus frutos se colectan en poblaciones silvestres, pero también existe manejo silvícola y cultivo de plantas de esta especie en sistemas agroforestales. Investigaciones previas documentaron divergencias morfofisiológicas y genéticas entre poblaciones silvestres y manejadas, ocasionadas por selección artificial y limitaciones al flujo génico entre poblaciones. En esto último podrían influir diferencias en patrones de germinación y establecimiento de semillas en plántulas silvestres y cultivadas en bosques y ambientes manejados. Se probó la hipótesis de que la selección artificial por frutos grandes favorece un mayor tamaño de las semillas en plantas cultivadas, las cuales presentarían mayor tasa de germinación en condiciones de alta humedad, pero serían más susceptibles a estrés hídrico que las semillas de plantas silvestres. Se evaluó el peso de frutos y semillas y su tasa de germinación en un gradiente de humedad $(0.0,-0.2,-0.4,-0.6,-0.8$ y -1.0 Megapascales) en poblaciones silvestres y cultivadas. Los frutos cultivados y sus semillas fueron significativamente más pesados $(54.616 \pm 1.547 \mathrm{~g}, 0.190 \pm 0.017 \mathrm{~g}$, respectivamente) que los frutos y semillas silvestres $(37.023 \pm$ $1.122 \mathrm{~g}, 0.153 \pm 0.005 \mathrm{~g}$, respectivamente). Los análisis de devianza mostraron diferencias significativas en el inicio $\left(\mathrm{X}^{2}=1,639.0\right.$, $\mathrm{gl}=4 ; \mathrm{X}^{2}=236.31, \mathrm{gl}=1$, respectivamente) y la velocidad de germinación $\left(\mathrm{X}^{2}=20.91, \mathrm{gl}=4\right)$, asociadas al tipo de manejo y al potencial hídrico (las semillas cultivadas fueron más susceptibles al estrés que las silvestres). La selección artificial que favorece frutos grandes parece haber influido indirectamente en el incremento de tamaño de las semillas, y las condiciones ambientales de los sitios manejados podrían haber influido en una mayor susceptibilidad a estrés hídrico.
\end{abstract}

Palabras clave: cactáceas columnares, domesticación, estrés hídrico, manejo tradicional, tamaño de semillas.

\begin{abstract}
Stenocereus stellatus is a columnar cactus species of cultural and ecological importance in the TehuacánValley; their fruits are collected from wild populations but plants of this species are also silviculturally managed and cultivated in agroforestry systems. Previous studies documented morpho-physiological and genetic divergences between wild and managed populations caused by artificial selection and limitations to gene flow; nevertheless, the documented divergences could also be influenced by differences in germination and survivorship patterns of wild and cultivated seedlings in forests and managed environments. We hypothesized that artificial selection in favor of larger fruits may in turn favor larger seeds in cultivated plants that also could have higher germination rates in high humidity conditions, but more susceptible to water stress than seeds from wild plants. We evaluated fruit and seed weight and germination rates in a water stress gradient $(0.0,-0.2,-0.4,-0.6,-0.8$, and -1.0 Megapascals $)$ in wild and cultivated populations. Cultivated fruits and seeds were significantly heavier $(54.616 \pm 1.547 \mathrm{~g}, 0.190 \pm 0.017 \mathrm{~g}$, respectively) than wild fruits and seeds $(37.023 \pm 1.122 \mathrm{~g}, 0.153 \pm 0.005 \mathrm{~g}$, respectively). Deviance analysis showed significant differences in starting germination $\left(X^{2}=1639.0, \mathrm{df}=4 ; \mathrm{X}^{2}=236.31, \mathrm{df}=1\right.$, respectively) and velocity $\left(X^{2}=20.91, \mathrm{df}=4\right)$ associated to management and water potential (cultivated seeds were more susceptible to water stress than wild ones). Artificial selection favoring larger fruits has apparently influenced indirectly seed size and differences; in wild and managed environments could have influenced differences of seed susceptibility to water stress.
\end{abstract}

Key words: columnar cacti, domestication, seed size, traditional management, water stress.

$\mathbf{L}$ a domesticación es un proceso evolutivo dirigido por seres humanos que resulta de la continua manipulación de la variación morfológica y fisiológica de poblaciones de plantas y animales con el fin de satisfacer necesidades culturales (Hawkes, 1983; Harlan, 1992; Casas et al., 1997). Se desarrolla principalmente por medio de selección artificial, favoreciendo la supervivencia y reproducción de feno- tipos convenientes a los seres humanos y desfavoreciendo o incluso eliminando otros no convenientes (Darwin, 1859; Harlan, 1992). Este proceso determina cambios en las frecuencias de los fenotipos y genotipos que componen las poblaciones de organismos manejados, con respecto a las que existen en poblaciones silvestres (Casas et al., 2007; Parra et al., 2010). Entre los cambios asociados a la selección ar- 
tificial en plantas destacan un mayor tamaño y diversidad morfológica de las partes útiles de la planta, cambios en la arquitectura, en la forma de vida, en sistemas reproductivos y en procesos ecofisiológicos como pérdida de latencia en las semillas y mayor velocidad y sincronía de la germinación (Evans, 1993). Algunos de estos cambios involucran pérdida de mecanismos de protección contra herbívoros o la pérdida de mecanismos naturales de reproducción, lo que resta a las plantas domesticadas capacidad de sobrevivir y reproducirse en ambientes silvestres (Hawkes, 1983; Harlan, 1992; Evans, 1993; Frary y Doğanlar, 2003), en donde las condiciones ecológicas suelen ser muy distintas a las de los ambientes cultivados.

Varios estudios han analizado cómo operan los procesos de domesticación en especies de cactáceas columnares del Valle de Tehuacán, que es una de las áreas del continente Americano donde se han registrado los restos más antiguos de agricultura y domesticación de plantas (MacNeish, 1967). Estudios arqueológicos realizados en la zona (MacNeish, 1967) identificaron que desde la prehistoria los pueblos de la región han usado de forma excepcionalmente importante las cactáceas columnares, debido a su abundancia y diversidad, y a que la mayor parte de las especies de este grupo de plantas producen frutos comestibles (Casas y Barbera, 2002). En la actualidad, las cactáceas columnares son de los elementos más importantes desde el punto de vista ecológico, cultural y económico en la región (Casas y Barbera, 2002). Una de las especies de cactáceas columnares primeramente estudiadas, en el marco de la domesticación, ha sido Stenocereus stellatus, comúnmente conocida como "xoconochtli". Esta especie es endémica del centro de México, distribuyéndose en el Valle de Tehuacán y en la Depresión del río Balsas, en los estados de Morelos, Puebla, Guerrero y Oaxaca (Casas et al., 1997). Esta especie tiene diferentes usos, pero el más importante es el consumo de sus frutos (Casas et al., 1997; 1999a, b).

Estudios etnobotánicos (Casas et al., 1997) identificaron en Stenocereus stellatus tres tipos de manejo: (1) recolección de frutos directamente en las poblaciones silvestres; (2) manejo in situ o silvícola, que consiste en que durante el aclareo de áreas forestales para establecer campos de cultivo se dejan en pie los individuos de ésta y otras especies en sistemas agroforestales; generalmente se mantienen aquellas plantas cuyos frutos presentan los mejores atributos para consumo humano (mayor tamaño, sabor dulce, entre otras características, como se detalla adelante); y (3) se cultiva en huertos y/o solares (Casas et al., 1997), generalmente se propagan las partes vegetativas de individuos con los mejores atributos. Estudios morfométricos (Casas et al., 1999a) documentaron que los frutos son el principal blanco de selección artificial y que hay divergencias morfológicas significativas entre las poblaciones silvestres y las manejadas (bajo manejo silvícola y cultivadas). En las poblaciones cultivadas hay una mayor proporción de individuos que producen frutos de mayor tamaño, con sabor más dulce, menos espinas, peri- carpio más delgado y con mayor proporción de variedades con pulpa distinta al color rojo (anaranjado, amarillo, rosado y morado), que prevalece en las poblaciones silvestres (Casas et al., 1999a). Estudios de genética de poblaciones (Casas et al., 2006; Cruse-Sanders et al., 2013) identificaron pequeñas, aunque significativas, distancias genéticas entre poblaciones; sin embargo, tales distancias se correlacionan con la distancia geográfica más que con el tipo de manejo. Patrones similares de divergencias morfológicas y genéticas se han documentado en otras especies de cactáceas columnares del Valle de Tehuacán-Cuicatlán que reciben manejo tradicional similar a Stenocereus stellatus; tales son los casos de S. pruinosus (Luna-Morales y Aguirre, 2001; Parra et al., 2008, 2010, 2012), P. chichipe (Otero-Arnaiz et al., 2003; 2005a, b; Carmona y Casas, 2005), Lemairocereus hollianus (Rodríguez-Arévalo et al., 2006) y Myrtillocactus schenckii (Blancas et al., 2009), y aun en especies que solamente reciben manejo silvícola y no se cultivan en huertas, tales como Polaskia chende (Cruz y Casas, 2002) y Escontria chiotilla (Arellano y Casas, 2003; Tinoco et al., 2005).

El grado de diferenciación morfológica y genética entre las poblaciones silvestres y manejadas está asociado a la intensidad con la que operan la selección artificial en poblaciones manejadas y la selección natural en poblaciones silvestres (Casas et al., 2007). También se ha propuesto que en el proceso de diferenciación debe influir en procesos que limitan el flujo génico entre poblaciones silvestres y manejadas. Casas et al. (1999b), por ejemplo, investigaron posibles diferencias en la biología de la polinización que pudieran constituir barreras significativas al flujo de polen. Encontraron que la producción temporal de flores, la biología floral y el sistema de cruzamiento son similares en las poblaciones silvestres y manejadas, y que el flujo de polen puede ocurrir a distancias mayores que las que las separan, debido a la participación de murciélagos en la polinización (Casas et al., 1999b). Sin embargo, Arias-Cóyotl et al. (2006) encontraron diferencias en las especies de polinizadores que visitan las flores de poblaciones silvestres y cultivadas, lo que podría indicar la existencia de limitaciones parciales al flujo de polen. Por otro lado, el continuo recambio de materiales que practican los campesinos también influye en la diferenciación entre las poblaciones manejadas in situ y las cultivadas. Además, se ha identificado que algunos fenotipos favorables para la gente aparentemente son incapaces de sobrevivir en condiciones silvestres y sólo logran hacerlo bajo condiciones de cuidado humano (Casas et al., 1999b). En consecuencia, Casas et al. (1999b) propusieron que el éxito diferencial en la germinación de semillas y en la supervivencia de plántulas en distintos ambientes podría favorecer la divergencia entre poblaciones silvestres y domesticadas. Esto es, que las semillas y plántulas derivadas de poblaciones cultivadas pueden germinar, crecer y establecerse exitosamente en ambientes cultivados pero no en ambientes silvestres. Estas diferencias podrían ser explicadas en parte 
por diferencias en el tamaño de las semillas que influye en el vigor de las plántulas debido a características morfo-anatómicas que se expresan durante las primeras fases del desarrollo. Tales diferencias podrían determinar capacidades diferenciales de establecimiento exitoso bajo condiciones ambientales específicas, como lo ha sugerido el estudio de Loza-Cornejo y Terrazas (2011) con varias especies de cactáceas columnares. Estos factores, en estricto sentido, sugieren que a la par de la selección artificial por frutos (y semillas, indirectamente) podría operar la selección natural de manera distinta en ambientes silvestres y cultivados. Si esto está ocurriendo, el mecanismo podría aportar una explicación adicional a la abundancia de fenotipos convenientes a los humanos en los ambientes antropogénicos.

Rojas-Aréchiga et al. (2001) y Otero-Arnaiz et al. (2003) realizaron estudios de germinación de semillas provenientes de poblaciones manejadas y silvestres de Stenocereus stella- tus y Polaskia chichipe, respectivamente; encontraron que las semillas de las poblaciones manejadas germinaron en mayor porcentaje y velocidad que las de poblaciones silvestres. Sin embargo, estos experimentos probaron las diferencias sólo en condiciones óptimas de humedad. Guillén et al. $(2009,2011)$ estudiaron cinco especies de cactáceas columnares ubicadas en un gradiente de menor a mayor intensidad de manejo (Escontria chiotilla, Myrtillocactus schenckii, $P$. chende, P. chichipe y $S$. pruinosus), comparando los patrones de germinación de semillas de poblaciones silvestres y manejadas (silvícolas y cultivadas), en distintas condiciones de humedad que simulan ambientes silvestres y cultivados. Encontraron que las semillas de las poblaciones manejadas son más grandes y tienen mayores tasas de germinación, pero también son más susceptibles en condiciones de estrés hídrico que las semillas de poblaciones silvestres.

El presente estudio estuvo dirigido a investigar la rela-

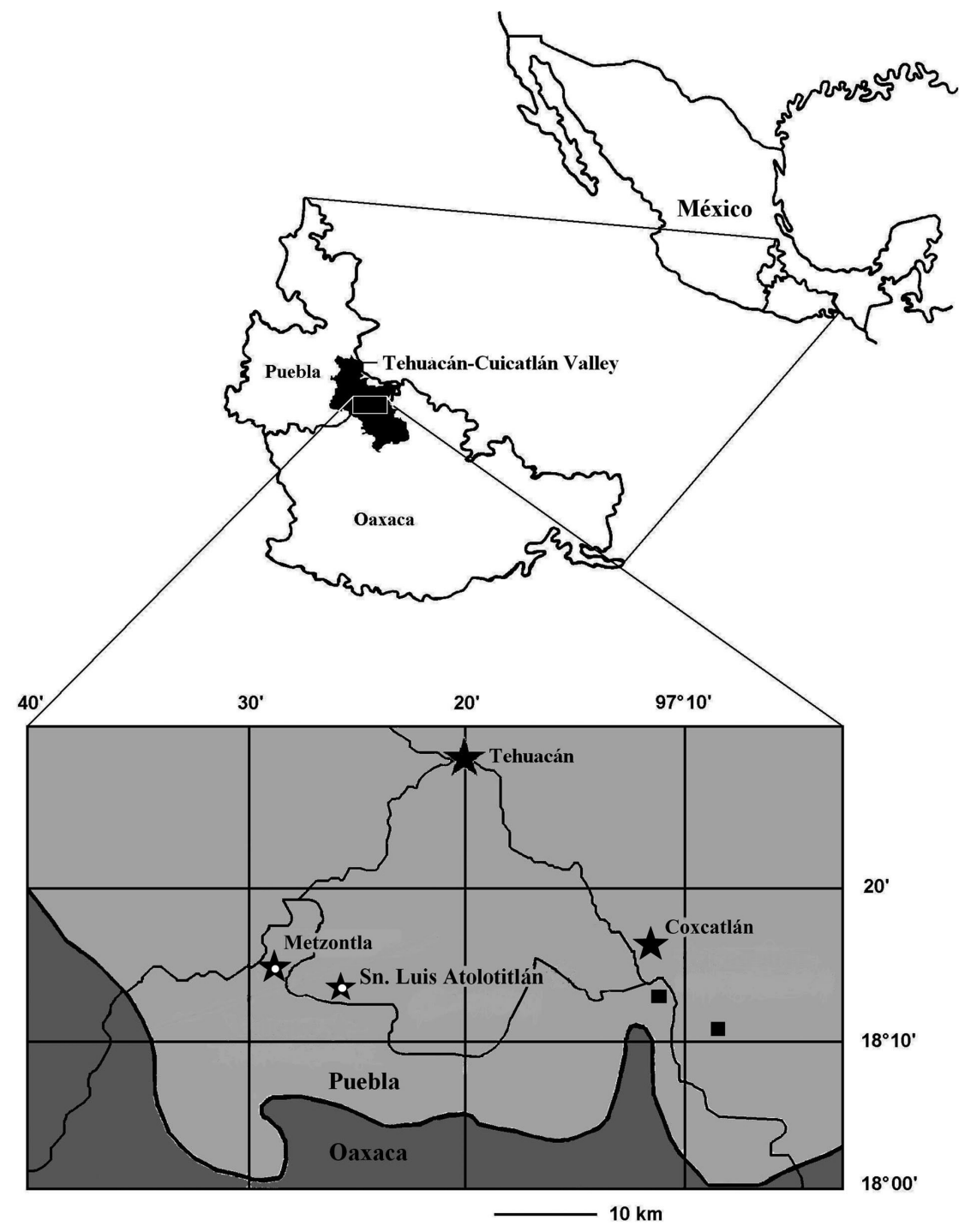

Figura 1. Área de estudio, municipios en los que fueron colectados frutos silvestres ( $\square$ ) y cultivados (O) de Stenocereus stellatus, dentro de la Reserva de la Biósfera de Tehuacán-Cuicatlán, México. 
ción entre tamaño de frutos y semillas de Stenocereus stellatus y los patrones de germinación de éstas últimas en un gradiente de estrés hídrico. Con base en los estudios de germinación de otras especies de cactáceas columnares, se planteó como hipótesis que la selección artificial a favor de frutos de mayor tamaño en $S$. stellatus tiene efecto en el tamaño de las semillas, lo que a su vez presentaría mayores tasas de germinación en condiciones de alta disponibilidad de humedad; sin embargo, se esperaría que éstas fueran más susceptibles al estrés hídrico que las semillas más pequeñas de las poblaciones silvestres. Los objetivos de este estudio fueron: (1) analizar las diferencias del tamaño de los frutos y de las semillas y si éstos difieren significativamente entre poblaciones silvestres y manejadas (manejadas in situ y cultivadas), y (2) analizar si las diferencias en tamaño se relacionan con diferencias en el patrón de germinación en un gradiente de estrés hídrico.

\section{Materiales y métodos}

Área de estudio. Se estudiaron dos poblaciones silvestres y dos cultivadas de Stenocereus stellatus. Las poblaciones silvestres forman parte del bosque tropical deciduo, en suelos aluviales en los que $S$. stellatus es uno de los componentes dominantes de la vegetación junto con otras especies de cactáceas columnares, como Lemairocereus hollianus y Pachycereus weberi (Casas et al., 1999a). Las poblaciones cultivadas estudiadas se encuentran en San Luis Atolotitlán y Los Reyes Metzontla, Puebla, en donde el clima es semiárido con temperatura y precipitación media anual de $18{ }^{\circ} \mathrm{C}$ y $655 \mathrm{~mm}$, respectivamente, de acuerdo con los datos registrados en la estación meteorológica más cercana, en Caltepec, Puebla (García, 1988). Ambas poblaciones silvestres se localizan en el municipio San Rafael Coxcatlán, próximas al sitio "Cueva del Maíz" donde la temperatura y la lluvia media anual son $24^{\circ} \mathrm{C}$ y $441 \mathrm{~mm}$, respectivamente (Casas et al., 1999b). Los sitios estudiados están dentro de la Reserva de la Biósfera Tehuacán-Cuicatlán, al sureste del estado de Puebla (Figura 1).

Colecta de frutos. En septiembre de 2009 se colectaron frutos de diez individuos en cada población silvestre y cultivada. De cada individuo se colectaron de 5 a 8 frutos maduros; cada fruto se pesó inmediatamente después de ser colectado. Todos los frutos colectados estaban dentro de la amplitud de peso promedio \pm una desviación estándar reportada en estudios morfométricos previos para poblaciones silvestres y cultivadas de la misma especie (Casas et al., 1999b). Esta estrategia de muestreo se llevó a cabo con el objetivo de prevenir los efectos asociados a variaciones extremas en los fenotipos presentes en las poblaciones estudiadas. Posteriormente se mezclaron semillas de diferentes frutos de cada tipo de población y se formaron lotes de 100 semillas y se pesaron. Las semillas se almacenaron a temperatura ambiente $\left(20 \pm 2{ }^{\circ} \mathrm{C}\right)$ en bolsas de papel durante 14 meses antes de iniciar los experimentos de germinación; esto se hizo tomando en cuenta estudios previos (Rojas-Aréchiga et al., 2001; Godínez-Álvarez et al., 2005) que reportan que las semillas de S. stellatus requieren un periodo de almacenamiento para germinar. Los experimentos de germinación se hicieron con mezclas de semillas de los diferentes frutos colectados para cada tipo de población.

Establecimiento de tratamientos con diferente disponibilidad de humedad. Para analizar el efecto del estrés hídrico sobre la velocidad y porcentaje de germinación se establecieron diferentes tratamientos de potencial hídrico, generados mediante soluciones de Polietilenglicol 8000 (PEG 8000, Sigma) a diferentes concentraciones. Las concentraciones se determinaron utilizando el programa Solute Potential and MolarMolal-Solute/g Water Interconversion (SPMM por sus siglas en inglés, Michel y Radcliffe, 1985). Las concentraciones usadas fueron, en Megapascales (MPa): (1) 0.0 (control, con agua destilada), (2) $-0.2 \mathrm{MPa}[0.021 \mathrm{M}]$, (3) - $0.4 \mathrm{MPa}[0.028$ M], (4) -0.6 MPa [0.033 M], (5) -0.8 MPa [0.037 M] y - 1.0 MPa [0.041 M]. En cajas Petri $(100 \times 15 \mathrm{~mm})$ se vertieron 20 ml de solución con PEG y se colocaron 25 semillas sobre una gasa de tela estéril con la finalidad de evitar que se sumergieran en la solución. Las cajas Petri fueron selladas e incubadas en una cámara de germinación (Enviromental Growth Chambers) que se mantuvo a una temperatura constante de $25^{\circ} \mathrm{C}$ y a un fotoperiodo neutro de $12 \mathrm{~h}$ de luz. Cada tratamiento estuvo compuesto por cuatro réplicas.

Antes de realizar el experimento, las semillas fueron desinfectadas sumergiéndolas por 15 min en una solución de hipoclorito de sodio al $70 \%$. El criterio utilizado para considerar una semilla como germinada fue la observación de la radícula. Se evaluó la germinación cada 24 h durante 40 días.

\section{Análisis estadísticos.}

Peso de los frutos y las semillas.- Las diferencias en los pesos de los frutos y de los lotes de semillas entre poblaciones se analizaron por medio de análisis de varianza (ANOVA) de una vía, evaluando el efecto de la variable categórica Manejo con dos niveles (Silvestres y Cultivadas). Se utilizó el programa estadístico $R$ (versión 2.13 2, R Development Core Team, 2008).

Evaluación de la germinación.- La germinación máxima fue definida como el porcentaje acumulativo de germinación después de 40 días a partir de la fecha en que las semillas fueron sembradas. El efecto del tipo de manejo y de potencial hídrico sobre la tasa de germinación (porcentaje de germinación por día), se evaluó por medio de un análisis de devianza con base en un Modelo Lineal Generalizado (Crawley, 1993). La proporción acumulativa de semillas germinadas fue la variable dependiente; el tiempo (días a partir de la fecha en que las semillas fueron sembradas), fue la variable continua independiente; el potencial hídrico (factor con seis niveles) y el tipo de manejo (factor con dos 
Cuadro 1. Peso $(\mathrm{g}) \pm$ Error estándar de frutos y semillas de poblaciones silvestres y cultivadas de Stenocereus stellatus. Resultados de ANOVA de una vía del peso de frutos (78 silvestres y 98 cultivados) y semillas (30 lotes de 100 semillas).

\begin{tabular}{lrrr}
\hline \multicolumn{3}{c}{ Silvestre } & \multicolumn{1}{c}{ Cultivada } \\
\hline Frutos & $37.023 \pm 1.122$ & $54.616 \pm 1.547$ & $F_{(1,174)}=77.206 ; P<0.001$ \\
Semillas & $0.153 \pm 0.005$ & $0.190 \pm 0.017$ & $F_{(1,58)}=4.250 ; P<0.05$ \\
\hline
\end{tabular}

niveles) fueron las variables categóricas independientes. Se utilizó un error binomial, los datos se ajustaron a una función logística como se indica para variables dependientes proporcionales; en este tipo de error, la devianza (equivalente a la varianza en los modelos con error de tipo normal) explicada por las variables independientes puede ser considerada como una aproximación al valor de $\mathrm{X}^{2}$ (Crawley, 1993). La proporción acumulativa de semillas que germinaron $(y)$ después de t días fue dada por el siguiente modelo:

$$
y=e\left\{[a+(b t)]-\left(c t^{2}\right)\right\} / 1+e\left\{[a+(b t)]-\left(c t^{2}\right)\right\}
$$

Donde $\boldsymbol{a}$ es la ordenada al origen que indica el inicio de la germinación, $\boldsymbol{b}$ es la tasa de germinación inicial, $\boldsymbol{c}$ indica el incremento de la tasa de germinación (en este caso $\boldsymbol{c}$ adquiere valores negativos) o el decremento (en este caso $c$ adquiere valores positivos) a través del tiempo. El efecto de los tratamientos de manejo y potencial hídrico sobre la tasa de germinación fue evaluada por la devianza explicada por la interacción de cada factor o ambos, con el tiempo linear o el cuadrático $\left(t^{2}\right)$. Los análisis fueron realizados con el programa estadístico GLIM versión 3.77 (Royal Statistical Society, 1985).

\section{Resultados}

Peso de frutos y semillas. Los frutos y semillas, de las poblaciones cultivadas de Stenocereus stellatus, tuvieron un peso significativamente mayor respecto a los frutos y las semillas de poblaciones silvestres (Cuadro 1).

Tasa de germinación. En casi todos los tratamientos de disponibilidad de humedad que se probaron $(0.0,-0.2,-0.4$, -0.6 y $-0.8 \mathrm{MPa}$ ), las semillas silvestres germinaron en ma- yor porcentaje que las cultivadas (Figura 2). A lo largo de 40 días se registró germinación de semillas de poblaciones silvestres en el gradiente de potencial hídrico de 0.0 a -0.8 $\mathrm{MPa}$, mientras que las semillas de poblaciones cultivadas sólo germinaron en el gradiente de 0.0 a $-0.4 \mathrm{MPa}$ (Figura 2). En el tratamiento de menor disponibilidad de humedad $(-1.0)$ no germinaron ni las semillas de plantas silvestres ni las de plantas cultivadas.

El modelo logístico ajustado explica el $96 \%$ de la variación de la germinación a través del tiempo. Los factores Potencial hídrico al inicio de la germinación y Tiempo explican un alto porcentaje de la varianza total (66 y $15 \%$, respectivamente); ambos factores fueron altamente significativos, lo que indica que la disponibilidad de humedad afectó el inicio de la germinación y que hay diferencias en las pendientes de las curvas de germinación. Esto último está relacionado con la velocidad de germinación (Cuadro 2, Figura 2). Además, al ser significativo en el modelo, el factor Manejo indica que el inicio de la germinación fue diferente entre semillas silvestres y cultivadas en todo el gradiente de humedad (Cuadro 2, Figura 2). En el tratamiento control se registraron los mayores porcentajes de germinación (68.85 y $41.23 \%$ silvestres y cultivadas, respectivamente). En este tratamiento, las semillas silvestres empezaron a germinar al cuarto día después de iniciado el experimento y las cultivadas al sexto. Conforme disminuyó la disponibilidad de humedad el inicio de la germinación se fue retrasando (Figura 2A). El Tiempo cuadrático $\left(\right.$ Tiempo $^{2}$ ) fue significativo, lo que indica que la tasa de germinación declina al avanzar el tiempo. La interacción Tiempo $\times$ manejo fue significativo (Cuadro 2, Figura 2), e indica que las pendientes de las curvas que describen la tasa de germinación de semillas silvestres y cultivadas variaron significativamente a través del tiempo.

Cuadro 2. Análisis de devianza del número de semillas germinadas de poblaciones silvestres y cultivadas, en un gradiente de disponibilidad de humedad de la cactácea columnar Stenocereus stellatus.

\begin{tabular}{lllll}
\hline Factor & Devianza $\left(\mathbf{X}^{2}\right)$ & gl & $\mathbf{r}^{2}$ & $\boldsymbol{P}$ \\
\hline Manejo & 236.31 & 1 & 0.095 & $<0.001$ \\
Potencial Hídrico & $1,639.00$ & 4 & 0.661 & $<0.001$ \\
Manejo $\times$ Potencial Hídrico & 20.91 & 4 & 0.008 & $<0.001$ \\
Tiempo & 376.70 & 1 & 0.151 & $<0.001$ \\
Tiempo & 118.24 & 1 & 0.047 & $<0.001$ \\
Tiempo $\times$ Manejo & 8.60 & 1 & 0.003 & $<0.01$ \\
Modelo & $2,399.76$ & 12 & 0.968 & 0.031 \\
Residual & 79.06 & 387 & & \\
Total & $2,478.82$ & 399 & & \\
\hline
\end{tabular}



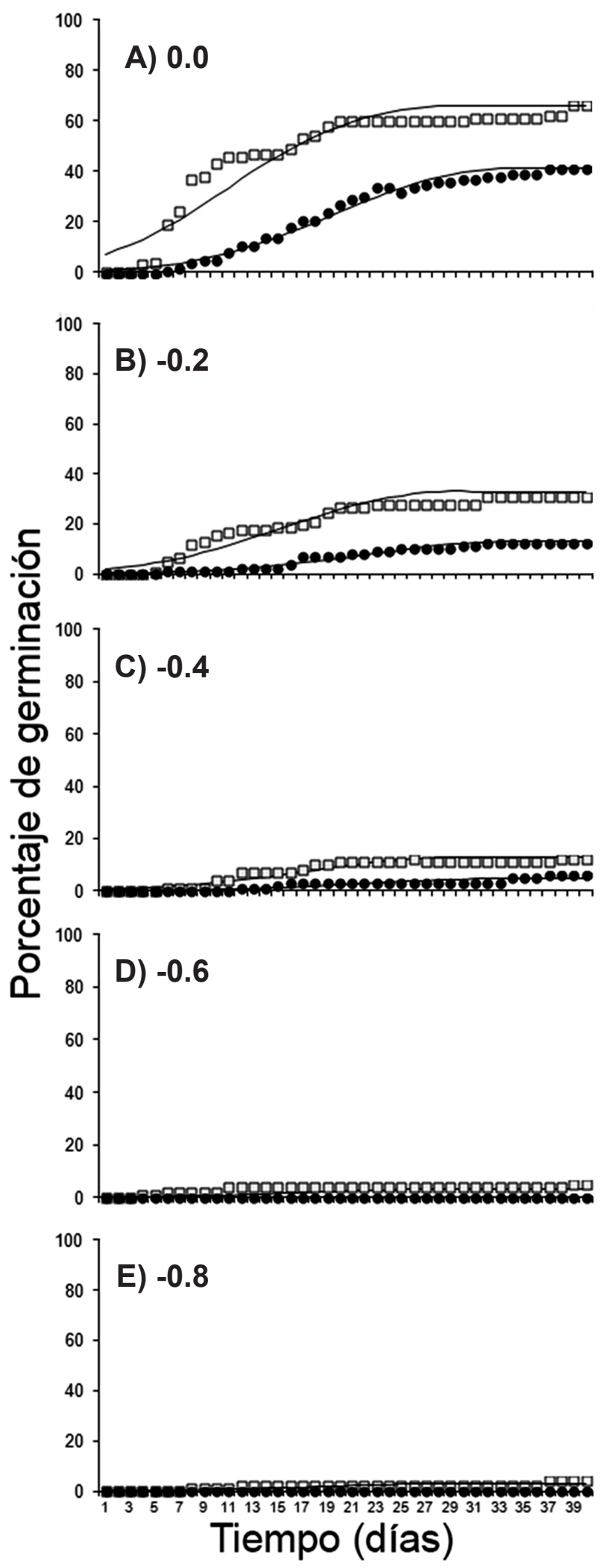

Figura 2. Modelos Log-lineares de germinación de semillas de poblaciones silvestres $(\square)$ y cultivadas $(\boldsymbol{O})$ de Stenocereus stellatus en diferentes tratamientos de potencial hídrico, generados mediante soluciones de PEG 8000.

\section{Discusión}

En Stenocereus stellatus, como en otras especies de árboles frutales, los principales blancos de selección artificial son atributos de los frutos (Casas et al., 1997, 1999a, b). La gente que aprovecha esta especie selecciona los frutos de su preferencia durante la recolección en poblaciones silvestres, y esta actitud selectiva se extiende como criterio para dejar en pie o para propagar ramas de aquellos individuos que presentan las mejores características. Prefieren a los que producen los frutos más grandes, con sabor más dulce, cáscara (pericarpio) delgada y con menos espinas (Casas et al., 1997). Este estudio corrobora que tanto los frutos como las semillas de poblaciones cultivadas de $S$. stellatus tuvieron mayor peso que los de las poblaciones silvestres, lo que coincide con información reportada para ésta y otras especies de cactáceas columnares (Casas et al., 1999b; RojasAréchiga et al., 2001; Guillén et al., 2011). Los frutos de plantas cultivadas de $S$. stellatus son en promedio $60 \%$ más pesados que los silvestres. En la actualidad, el peso de las semillas no es un carácter directamente seleccionado por la gente; pudo haberlo sido en el pasado, pues estudios etnobotánicos han reportado que en algunas regiones del Valle de Tehuacán las semillas son usadas en la elaboración de salsas tradicionales, y por tanto, tienen cierto valor económico, un litro de semilla se intercambia por 15 litros de maíz (Casas et al., 1997). Sin embargo, no existe un registro claro de que en el presente se lleve a cabo selección artificial a favor de este carácter. El mayor tamaño de semillas registrado en los frutos cultivados puede explicarse como una consecuencia de la selección artificial dirigida a frutos de mayor tamaño, los cuales también presentan una mayor cantidad de pulpa, que a su vez está relacionada con la cantidad y el tamaño de los funículos (Casas et al., 1999a).

Se registraron bajas tasas de germinación; resultados que coinciden con los porcentajes de germinación ya reportados para la misma especie (Rojas-Aréchiga et al., 2001; Godínez-Álvarez et al., 2005), los cuales sugieren que las semillas de Stenocereus stellatus necesitan un tiempo de almacenamiento para incrementar la tasa de germinación. En el presente estudio, las semillas de las mismas muestras no germinaron después de tres meses de almacenamiento, y sí lo hicieron después de 14 meses. De acuerdo con RojasAréchiga et al. (2001), se espera que estas mismas muestras aumenten su desempeño germinativo después de un mayor periodo de almacenamiento.

Los experimentos de germinación realizados en el presente estudio no coinciden con los resultados reportados previamente por Rojas-Aréchiga et al. (2001) para la misma especie en condiciones de máxima disponibilidad de humedad. Estos autores, como se mencionó anteriormente, registraron que las semillas de poblaciones cultivadas germinaron en mayor porcentaje que las de poblaciones silvestres. Cabe señalar que las semillas utilizadas por Rojas-Aréchiga et al. 
(2001) fueron más pequeñas (cultivadas $0.112 \pm 0.004 \mathrm{~g}$, silvestres $0.080 \pm 0.02 \mathrm{~g}$ ) que las semillas utilizadas en este trabajo (cultivadas $0.190 \pm 0.005 \mathrm{~g}$, silvestres $0.153 \pm 0.005 \mathrm{~g}$ ) y tuvieron diferente periodo de almacenamiento; por lo que los resultados no son necesariamente comparables. Sin embargo, al disminuir la disponibilidad de humedad se observó que las semillas silvestres fueron menos susceptibles que las cultivadas. Este es un resultado similar a lo registrado para otras especies de cactáceas columnares sometidas a gradientes similares de estrés hídrico (Guillén et al., 2011). Nuestros estudios previos (Guillén et al., 2009, 2011) sugieren que en cada especie hay un punto crítico en el gradiente de estrés hídrico en el que es posible apreciar las diferencias más marcadas entre las curvas de germinación de semillas silvestres y cultivadas. Además, los resultados indican que tal punto crítico es diferente para cada especie y que éste parece estar relacionado con adaptaciones particulares de cada especie al hábitat en el que se distribuyen (Guillén et al., 2011). En comparación con otras especies de cactáceas columnares estudiadas, en Stenocereus stellatus la germinación de semillas es susceptible a la falta de humedad, incluso más susceptible que las de S. pruinosus (Guillén et al., 2011). Por esta razón, aunque se han observado plántulas derivadas de semilla de esta especie en el Valle de Tehuacán (Godínez-Álvarez et al., 2005), es más común observar plantas producto de la propagación vegetativa y el reclutamiento sexual ocurre seguramente de manera episódica, durante años de precipitación pluvial excepcionalmente alta. En la Mixteca Baja, donde la precipitación es más alta que en Tehuacán, es más común observar la reproducción sexual de esta especie.

La continua selección por frutos de mayor tamaño parece haber tenido como consecuencia indirecta la producción de semillas de mayor tamaño y, en consecuencia, más susceptibles en condiciones de estrés hídrico. En ambientes cultivados esta característica puede no ser tan relevante en términos selectivos, pues en éstos existen condiciones mésicas que favorecen la germinación de semillas susceptibles a estrés hídrico. Sin embargo, en condiciones silvestres las diferencias en comportamiento germinativo sí son relevantes; es posible predecir que bajo condiciones silvestres la germinación y supervivencia de plántulas susceptibles a estrés hídrico sea significativamente menor que la de las semillas pequeñas. Tales diferencias parecen resultar, entonces, del efecto de selección artificial a favor de frutos de mayor tamaño, así como selección natural asociada a las condiciones ambientales en las que crecen las plantas silvestres y las manejadas. Asimismo, estas diferencias podrían contribuir a explicar las divergencias morfológicas, genéticas y reproductivas documentadas entre poblaciones silvestres y manejadas de esta especie.

La supervivencia y crecimiento de las plántulas también son aspectos críticos del ciclo de vida que pueden reflejar importantes condiciones diferenciales de la selección natural en condiciones silvestres y ambientes cultivados. Es necesario realizar estudios de la supervivencia y crecimiento diferencial de plántulas cultivadas y silvestres, en ambientes cultivados y silvestres para tener un mejor o mayor conocimiento acerca de los factores que explican la diferenciación morfo-fisiológica y genética de poblaciones silvestres y manejadas simpátricas de Stenocereus stellatus. Es también importante realizar estudios de germinación y establecimiento en campo para identificar otros factores que, además de la humedad, pueden influir en el establecimiento. El presente trabajo sugiere que el estudio de la germinación de semillas y establecimiento de plántulas es crucial para entender mecanismos que limitan el flujo génico entre poblaciones silvestres y cultivadas, y favorecen los procesos de divergencia que se han documentado en el proceso de domesticación de S. stellatus.

\section{Agradecimientos}

Los autores agradecen a dos revisores anónimos por la revisión del manuscrito y los comentarios que lo enriquecieron. Igualmente agradecemos apoyo financiero del Programa de Apoyo a proyectos de Investigación e Innovación Tecnológica (PAPIIT, proyecto IN205111-3) y del CONACYT (Proyecto CB-2008-01-103551). Igualmente reconocemos y agradecemos al Dr. Alejandro Martínez Palacios de la Universidad Michoacana de San Nicolás de Hidalgo por las facilidades en el uso de infraestructura para realizar los experimentos, así como a Edgar Pérez Negrón, Alberto Valencia y Heberto Ferreira por el apoyo técnico en la realización del trabajo y análisis de información.

\section{Literatura citada}

Arellano E. y Casas A. 2003. Morphological variation and domestication of Escontria chiotilla (Cactaceae) under silvicultural management in Tehuacán Valley, Central Mexico. Genetic Resources and Crop Evolution 50:439-453.

Arias-Cóyotl E., Stoner K.E. y Casas A. 2006. Effectiveness of bats as pollinators of Stenocereus stellatus (Cactaceae) in wild, managed in situ, and cultivated populations in La Mixteca Baja, central Mexico. American Journal of Botany 93:1675-1683.

Blancas J., Casas A., Lira R. y Caballero J. 2009. Traditional management and morphological patterns of Myrtillocactus schenckii (Cactaceae) in the Tehuacán Valley, Central Mexico. Economic Botany 63:375-387.

Carmona A. y Casas A. 2005. Management, phenotypic patterns and domestication of Polaskia chichipe (Cactaceae), in the Tehuacán Valley, Central México. Journal of Arid Environments 60:115-132.

Casas A. y Barbera G. 2002. Mesoamerican domestication and diffusion. En: Nobel P.S. Ed. Cacti: Biology and Uses, pp. 143162, University of California Press, Berkeley.

Casas A., Caballero J. y Valiente-Banuet A. 1999a. Use, management and domestication of columnar cacti in south-central México: a historical perspective. Journal of Ethnobiology 19:71-95.

Casas A., Otero-Arnaiz A., Pérez-Negrón E. y Valiente-Banuet 
A. 2007. In situ management and domestication of plants in Mesoamerica. Annals of Botany 100:1101-1115.

Casas A., Pickersgill B., Caballero J. y Valiente-Banuet A. 1997. Ethnobotany and domestication in xoconochtli, Stenocereus stellatus (Cactaceae), in the Tehuacán Valley and La Mixteca Baja, México. Economic Botany 51:279-292.

Casas A., Caballero J., Valiente-Banuet A., Soriano J.A. y Dávila P. 1999b. Morphological variation and the process of domestication of Stenocereus stellatus (Cactaceae) in Central Mexico. American Journal of Botany 86:522-533.

Casas A., Cruse-Sanders J., Morales E., Otero-Arnaiz A. y Valiente-Banuet A. 2006. Maintenance of phenotypic and genotypic diversity in managed populations of Stenocereus stellatus (Cactaceae) by indigenous peoples in Central Mexico. Biodiversity and Conservation 15:879-898.

Crawley M.J. 1993. GLIM for Ecologists. Blackwell Scientific Publications, Oxford.

Cruse-Sanders J.M., Parker K.C., Friar E.A., Huang D.I., Mashayekhi S., Prince L.M., Otero-Arnaiz A. y Casas A. 2013. Managing diversity: domestication and gene flow in Stenocereus stellatus Riccob. (Cactaceae) in Mexico. Ecology and Evolution 3:1340-1355.

Cruz M. y Casas A. 2002. Morphological variation and reproductive biology of Polaskia chende (Cactaceae) under domestication in Central Mexico. Journal of Arid Environments 51:561-576.

Darwin C. 1859. On the Origin of Species by Means of Natural Selection, or the Preservation of Favored Races in the Struggle for Life. Jonh Murray, Londres.

Evans L.T. 1993. Crop Evolution, Adaptation and Yield. Cambridge University Press, Cambridge.

Frary A. y Doğanlar S. 2003. Comparative genetics of crop plant domestication and evolution. Turkish Journal of Agriculture and Forestry 27:59-69.

García E. 1988. Modificaciones al Sistema de Clasificación Climática de Köppen (para Adaptarlo a las Condiciones de la República Mexicana). Instituto de Geografía, Universidad Nacional Autónoma de México. México, D.F.

Godínez-Álvarez H., Ríos-Casanova L. y Pérez F. 2005. Characteristics of seedling establishment of Stenocereus stellatus (Cactaceae) in the Tehuacán Valley, Mexico. The Southwestern Naturalist 50:375-380.

Guillén S., Benitez J., Martínez-Ramos M. y Casas A. 2009. Seed germination of wild, in situ-managed, and cultivated populations of columnar cacti in the Tehuacán-Cuicatlán Valley, Mexico. Journal of Arid Environments 73:407-413.

Guillén S., Terrazas T., De la Barrera E. y Casas A. 2011. Germination differentiation patterns of wild and domesticated columnar cacti in a gradient of artificial selection intensity. Genetic Resources and Crop Evolution 58:409-423.

Harlan J.R. 1992. Origins and processes of domestication. En: Chapman G.P. Ed. Grass Evolution and Domestication, pp. 159-175, Cambridge University Press, Cambridge.

Hawkes J.G. 1983. The Diversity of Crop Plants. Harvard University Press, Londres.

Loza-Cornejo S. y Terrazas T. 2011. Morfo-anatomía de plántulas en especies de Pachyceerae: ¿Hasta cuándo son plántulas? Boletín de la Sociedad Botánica de México 88:1-13.

Luna-Morales C. y Aguirre R.J.R. 2001. Clasificación tradicional, aprovechamiento y distribución ecológica de la pitaya mixteca en México. Interciencia 26:18-24.

MacNeish R.S. 1967. A summary of the subsistence. En: Byers D.S. Ed. The Prehistory of the Tehuacán Valley. Vol. 1: Environment and Subsistence, 209-231, University of Texas Press, Austin.

Michel B.E. y Radcliffe D. 1985. A computer program relating solute potential to solution composition for five solutes. Agronomy Journal 87:126-130.

Otero-Arnaiz A., Casas A. y Hamrick L. 2005a. Direct and indirect estimates of gene flow among wild and managed populations of Polaskia chichipe, an endemic columnar cactus in Central Mexico. Molecular Ecology 14:4313-4322.

Otero-Arnaiz A., Casas A., Hamrick J.L. y Cruse-Sanders J. 2005b. Genetic variation and evolution of Polaskia chichipe (Cactaceae) under domestication in the Tehuacán Valley, central Mexico. Molecular Ecology 14:1603-1611.

Otero-Arnaiz A., Casas A., Bartolo C., Pérez-Negrón E. y Valiente-Banuet A. 2003. Evolution of Polaskia chichipe (Cactaceae) under domestication in the Tehuacán Valley, Central México: Reproductive biology. American Journal of Botany 90:593-602.

Parra F., Blancas J. y Casas A. 2012. Landscape management and domestication of Stenocereus pruinosus (Cactaceae) in the Tehuacán Valley: human guided selection and gene flow. Journal of Ethnobiology and Ethnomedicine 8:32.

Parra F., Pérez-Nasser N., Lira R., Pérez-Salicrup D. y Casas A. 2008. Population genetics and process of domestication of Stenocereus pruinosus (Cactaceae) in the Tehuacán Valley, México. Journal of Arid Environments 72:1997-2010.

Parra F., Casas A., Peñaloza-Ramírez J.M., Cortés-Palomec A.C., Rocha-Ramírez V. y González-Rodríguez A. 2010. Evolution under domestication: ongoing artificial selection and divergence of wild and managed Stenocereus pruinosus (Cactaceae) populations in the Tehuacán Valley, Mexico. Annals of Botany 106:483-496.

R Development Core Team. 2008. R: A Language and Environment for Statistical Computing. R Foundation for Statistical Computing, Vienna.

Rodríguez-Arévalo I., Casas A., Lira R y Campos J.. 2006. Uso, manejo y proceso de domestication de Pachycereus hollianus (F.A.C. Weber) Buxb. (Cactaceae) en el Valle de Tehuacán-Cuicatlán, México. Interciencia 31:677- 685.

Rojas-Aréchiga M., Casas A. y Vázquez-Yañes C. 2001. Seed germination on wild and cultivated Stenocereus stellatus (Cactaceae) from the Tehuacán-Cuicatlán Valley, Central Mexico. Journal of Arid Environments 49:279-287.

Royal Statistical Society. 1985. GLIM (General Linear Interactive Model), versión 3.77. Royal Statistical Society, Londres.

Tinoco A., Casas A., Luna R. y Oyama K. 2005. Population genetics of Escontria chiotilla in wild and silvicultural managed populations in the Tehuacán Valley, Central México. Genetic Resources and Crop Evolution 52:525-538.

Recibido: 22 de noviembre de 2012

Aceptado: 9 de marzo de 2013 